\title{
Severe outcomes of COVID-19 among patients with COPD and asthma
}

\author{
Erik Soeren Halvard Hansen (10 ${ }^{1}$, Amalie Lykkemark Moeller ${ }^{2}$, Vibeke Backer ${ }^{1}$, \\ Mikkel Porsborg Andersen (10) ${ }^{2}$, Lars Kober $\mathbb{1}^{3}$, Kristian Kragholm ${ }^{4}$ and \\ Christian Torp-Pedersen ${ }^{2}$
}

Affiliations: ${ }^{1}$ Centre for Physical Activity Research, Rigshospitalet, University of Copenhagen, Copenhagen, Denmark. ${ }^{2}$ Dept of Clinical Research, Nordsjaellands Hospital, Hillerød, Denmark. ${ }^{3}$ Dept of Cardiology, Rigshospitalet, University of Copenhagen, Copenhagen, Denmark. ${ }^{4}$ Unit of Clinical Epidemiology and Biostatistics, and Dept of Cardiology, Aalborg University Hospital, Aalborg, Denmark.

Correspondence: Erik Soeren Halvard Hansen, Rigshospitalet 7641, The Centre for Physical Activity Research, Blegdamsvej 9, DK-2100, Copenhagen, Denmark. E-mail: eriksorenhalvardhansendagmail.com

\section{ABSTRACT}

Introduction: Patients with obstructive lung diseases are possibly at risk of developing severe outcomes of coronavirus disease 2019 (COVID-19). Therefore, the aim of this study was to determine the risk of severe outcomes of COVID-19 among patients with asthma and COPD.

Methods: We performed a nationwide cohort study of patients with COVID-19 from 1 February to 10 July 2020. All patients with COVID-19 registered in the Danish registers were included. Using International Classification of Diseases (ICD) codes and medication history, patients were divided into asthma, COPD or no asthma or COPD. Primary outcome was a combined outcome of severe COVID-19, intensive care or death.

Results: Out of 5104 patients with COVID-19 (median age 54.8 years (25-75th percentile 40.5 to 72.3 ); women, 53.0\%), 354 had asthma and 432 COPD. The standardised absolute risk of the combined endpoint was $21.2 \%$ (95\% CI 18.8-23.6) in patients with COPD, $18.5 \%$ (95\% CI 14.3-22.7) in patients with asthma and $17.2 \%$ (95\% CI 16.1-18.3) in patients with no asthma or COPD. Patients with COPD had a slightly increased risk of the combined end-point compared with patients without asthma or COPD (risk difference 4.0\%; 95\% CI 1.3-6.6; $\mathrm{p}=0.003$ ). In age standardised analyses, there were no differences between the disease groups. Low blood eosinophil counts $\left(<0.3 \times 10^{9}\right.$ cells $\left.\cdot \mathrm{L}^{-1}\right)$ were associated with increased risk of severe outcomes among patients with COPD.

Conclusion: Patients with COPD have a slightly increased risk of developing severe outcomes of COVID-19 compared with patients without obstructive lung diseases. However, in age-standardised analysis, the risk difference disappears.

@ERSpublications

Patients with chronic obstructive pulmonary disease, but not asthma, have slightly increased risk of severe outcomes of \#COVID19 compared with patients without obstructive lung disease. However, in age-standardised analysis, the risk difference disappears. https://bit.ly/2HshNz8

Cite this article as: Hansen ESH, Moeller AL, Backer V, et al. Severe outcomes of COVID-19 among patients with COPD and asthma. ERJ Open Res 2021; 7: 00594-2020 [https://doi.org/10.1183/ 23120541.00594-2020].

This article has supplementary material available from openres.ersjournals.com

Data availability: All data relevant to the study are included in the article or uploaded as supplementary information. Data will be available immediately after publication to anyone who wishes to access the data with any purpose, with no end-date.

Received: 19 Aug 2020 | Accepted after revision: 14 Oct 2020

Copyright $\odot$ ERS 2021. This article is open access and distributed under the terms of the Creative Commons Attribution Non-Commercial Licence 4.0. 


\section{Introduction}

The coronavirus disease 2019 (COVID-19) pandemic is spreading across the globe infecting millions of people. Vulnerable patient groups infected with severe acute respiratory syndrome coronavirus 2 (SARS-CoV-2) are at risk of developing acute respiratory failure ultimately leading to death. It is estimated that $10-20 \%$ of the population have asthma or COPD worldwide $[1,2]$, and thus research into the effects of COVID-19 in these patient groups is critical. Persons with asthma and COPD are potentially more susceptible to severe outcomes of COVID-19, as viral infections affecting the upper or lower airways are some of the leading causes of admissions and exacerbations [3, 4]. Recent Asian and European studies have found that COPD is associated with severe outcomes of COVID-19 [5, 6]. However, similar data on patients with asthma are scarce, and evidence on risk factors and patient characteristics within asthma and COPD is lacking. In COPD, high levels of Type 2 inflammation (defined as elevated blood eosinophils) is associated with increased risk of exacerbations [7]. However, in COVID-19, Type 2 inflammation has been suggested as potentially being protective against adverse outcomes of COVID-19 [8]. As societies on lockdown begin to open up and measures of social distancing are loosened, it is becoming clear that SARS-CoV-2 will persist in the world as a threat to vulnerable patient groups for several years. This underlines the importance of identifying whether people with certain diseases are more prone to severe outcomes of COVID-19 than others. Therefore, through the Danish registries, we sought to investigate whether asthma and COPD are risk factors for severe outcomes of COVID-19. Further, we wanted to investigate whether eosinophilic inflammation was associated with frequency of severe outcomes of COVID-19.

\section{Methods}

We performed a retrospective cohort study investigating development of severe outcomes of COVID-19 in patients with COPD and asthma registered in the Danish healthcare system during the COVID-19 pandemic. Patients were included between 1 February 2020 and 10 July 2020. At the end of follow-up, $13015(0.2 \%)$ of the Danish population had tested positive for COVID-19. In Denmark, ethical approval is not necessary for retrospective studies. However, the study was approved by the data responsible institute (The Capital Region of Denmark - approval number P-2019-191). The authors vouch for the integrity of the data and the analyses.

\section{Data sources}

We used unique personal identifiers (encrypted CPR) from the Danish administrative registries to link pseudoanonymized data to individual persons. Thereby, data concerning diagnoses from outpatient and hospitalisation contacts, hospital procedures, prescription fills, civil and vital status, as well as income and education were collected. From the Danish registries, data on diagnoses from hospitalisations are available from 1978, and diagnoses from outpatient contacts are available from 1996; similarly, all data on procedures are available from 1996, and all prescriptions filled since 1995 can be assessed. The Danish registries have been described in detail previously [9].

\section{Study patients and COVID-19}

We included all Danish residents receiving a COVID-19 diagnosis (International Classification of Diseases, 10th edition (ICD-10): B342A, B972 and B972A). The index date was day of diagnosis/admission for COVID-19.

\section{Severe combined end-point of COVID-19}

We defined a severe combined end-point of COVID-19 which included diagnosis of COVID-19 with severe respiratory syndrome (ICD-10 code B972A), admission to an intensive care unit (ICU) or death, whichever came first.

\section{Asthma and COPD}

Asthma and COPD were primarily defined from ICD-10 codes J43-45. Patients with asthma who were not identified by ICD code for asthma, due to primary asthma management outside hospital, were defined as asthma based on prescribed medication if within the last year they had filled a minimum of two prescriptions of inhaled corticosteroids [10] or leukotriene receptor antagonists without concurrent use of long-acting muscarinergic antagonists (figure 1). Patients with COPD who were not identified by ICD code in a hospital setting for COPD were defined as having COPD based on prescribed medication if within the last year they had filled a minimum of two prescriptions of long-acting $\beta$-agonists or long-acting muscarinergic antagonists without concurrent use of inhaled corticosteroids within the last 12 months. Patients over 60 years with use of long-acting $\beta$-agonists in combination with inhaled corticosteroids were considered as having COPD. If a patient was diagnosed with both COPD and asthma, 
FIGURE 1 Flowchart of diagnoses in the study population. First, patients were selected based on International Classification of Diseases (ICD) codes. Second, we went through medication histories to identify patients with asthma or COPD without an ICD code. Patients identified by ICD codes did not necessarily use inhaled medication.

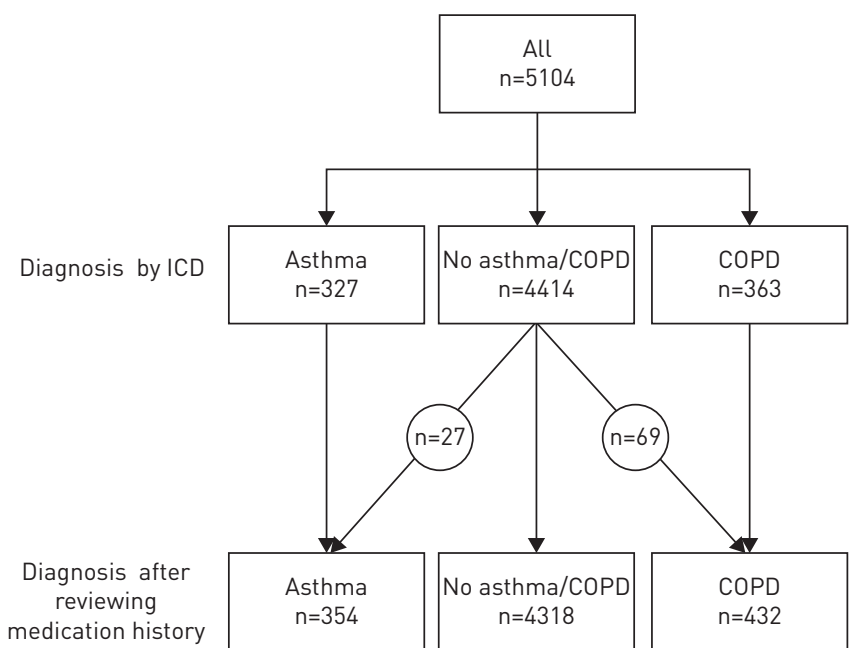

the patient was considered as having COPD. To compare our population to the general population, we estimated prevalence of COPD and asthma in the general population by the same criteria. All ICD-10 codes used are displayed in the supplementary material (Supplementary 1 and 2).

\section{Covariates}

We estimated current education level, household income, civil status defined as either "single" or "cohabiting" and origin defined as either "Danish" or "immigrant or descendants". Lastly, we calculated comorbidities from the last 10 years based on diagnosis from admissions and filled prescriptions. All definitions and ICD-10 codes for comorbidities are supplied in the supplementary material (Supplementary 1 and 2).

\section{Type 2 inflammation}

To evaluate Type 2 inflammation, we included blood eosinophils in our analysis. Blood eosinophil counts were collected from the last 5 years, and the most recent value was chosen for analysis. For analysis, values were factorised into $<0.3 \times 10^{9}$ and $\geqslant 0.3 \times 10^{9}$ cells $\cdot \mathrm{L}^{-1}$.

\section{Statistical considerations}

Comparisons of characteristics among included patients were analysed by the Chi-squared test for categorical variables or t-test for continuous variables. Our primary end-point was standardised absolute risk for the severe combined end-point at day 30 after first diagnosis of COVID-19 among patients with asthma, COPD or no obstructive lung disease. We calculated a standardised absolute risk at 30 days after COVID-19 diagnosis for the whole population by using g-formula methods based on multivariable Cox regression [11, 12].The Cox proportional hazards model was adjusted for age, sex, education level and a combined covariate for cardiac disease (heart failure, atrial fibrillation or flutter, or ischaemic heart disease) $[11,13,14]$. Variables and comorbidities included in the model were chosen based on that they could impact both the exposure and outcome and that they were available using a directed acyclic graph approach $[15,16]$ (see supplementary figure S4). To analyse the impact of increasing age on outcomes of COVID-19 in patients with asthma and COPD, we estimated the age-standardised absolute risk of the combined outcome at day 30 and its individual components. This analysis assumes that each patient with COVID-19 could have any age between 30 and 100 years and calculates the standardised absolute risk given that the patient had the assumed age. The applied Cox proportional hazards model was adjusted for sex, cardiac disease and education level. Lastly, in a complete-case analysis stratified by disease group, we estimated the standardised absolute risk of severe outcomes of COVID-19 at day 30 among patients with low and high levels of eosinophilia in their blood. We calculated an average absolute risk at 30 days after COVID-19 diagnosis for the whole population by using g-formula methods based on multivariable Cox regression $[11,12]$. Missingness was rare $(<1 \%)$; therefore, imputation methods were not necessary, and all analyses represent complete-case analysis.

All analyses were performed using R Core Team (2019) (The R Foundation, Vienna, Austria) [17]. 
Results

In this nationwide cohort study, we included 5104 patients with confirmed COVID-19 (table 1), 354 (6.9\%) of whom were defined as having asthma and 432 (8.5\%) as having COPD. When using the same diagnostic criteria in the general population we found that 90492/5920253 (1.5\%) had COPD and 270772 (4.6\%) had asthma. These results indicate that obstructive lung diseases are significantly over-represented in our sample population $(\mathrm{p}<0.001)$ (figure 1).

Patients with COPD were considerably older at admission compared with patients with asthma and patients without obstructive lung disease (median age 76.8, 47.5 and 53.0 years, respectively) ( $\mathrm{p}<0.001$ ). Level of education and mean household income were generally lower among patients with COPD

\section{TABLE 1 Characteristics of included coronavirus disease 2019 (COVID-19) population}

\begin{tabular}{|c|c|c|c|c|}
\hline Characteristics & Asthma & COPD & No asthma or COPD & p-value \\
\hline Subjects $\mathrm{n}$ & 354 & 432 & 4318 & \\
\hline Age median (IQR) & $47.5(24.1)$ & $76.8(13.7)$ & $53.0(30.0)$ & $<0.001$ \\
\hline Age groups & & & & $<0.001$ \\
\hline$<65$ years & $291(82.2)$ & 73 (16.9) & $3036(70.3)$ & \\
\hline $65-74$ years & $30(8.5)$ & 118 (27.3) & 495 (11.5) & \\
\hline$\geqslant 75$ years & 33 (9.3) & $241(55.8)$ & $787(18.2)$ & \\
\hline Female sex & 217 (61.3) & $220(50.9)$ & $2268(52.5)$ & \\
\hline Household income & & & & $<0.004$ \\
\hline Lowest quartile & 79 (22.3) & $154(35.6)$ & $1043(24.1)$ & \\
\hline Second lowest quartile & 75 (21.2) & $176(40.7)$ & $1025(23.7)$ & \\
\hline Second highest quartile & $95(26.8)$ & $75(17.4)$ & $1106(25.6)$ & \\
\hline Highest quartile & 105 (29.7) & $27(6.2)$ & $1144(26.5)$ & \\
\hline Highest completed education & & & & $<0.001$ \\
\hline Upper or lower secondary school & $85(24.0)$ & $188(43.5)$ & $1096(25.4)$ & \\
\hline Vocational upper secondary school & 108 (30.5) & $182(42.1)$ & $1616(37.4)$ & \\
\hline $\begin{array}{l}\text { Medium-cycle higher education or } \\
\text { bachelors' degree }\end{array}$ & 109 (30.8) & 49 (11.3) & $1100(25.5)$ & \\
\hline Long-cycle higher education & 52 (14.7) & $13(3.0)$ & 506 (11.7) & \\
\hline \multicolumn{5}{|l|}{ Civil status } \\
\hline Single & $116(32.8)$ & $217(50.2)$ & $1544(35.8)$ & $<0.001$ \\
\hline Married or cohabiting & $238(67.2)$ & $215(49.8)$ & $2774(64.2)$ & \\
\hline \multicolumn{5}{|l|}{ Origin } \\
\hline Danish & $281(79.4)$ & 392 (90.7) & 3518 (81.5) & $<0.001$ \\
\hline Immigrants or immigrants' descendants & $73(20.6)$ & $40(9.3)$ & $800(18.5)$ & \\
\hline \multicolumn{5}{|l|}{ Comorbidities } \\
\hline Ischaemic heart disease & $10(2.8)$ & $98(22.7)$ & $271(6.3)$ & $<0.001$ \\
\hline Previous myocardial infarction & $9(2.5)$ & $96(22.2)$ & $232(5.4)$ & $<0.001$ \\
\hline Heart failure & $7(2.0)$ & 83 (19.2) & $147(3.4)$ & $<0.001$ \\
\hline Atrial fibrillation or flutter & $27(7.6)$ & $139(32.2)$ & $474(11.0)$ & $<0.001$ \\
\hline Cerebral vascular disease & $13(3.7)$ & $91(21.1)$ & $276(6.4)$ & $<0.001$ \\
\hline Diabetes & $34(9.6)$ & 109 (25.2) & $455(10.5)$ & $<0.001$ \\
\hline Chronic kidney disease & $7(2.0)$ & $63(14.6)$ & $214(5.0)$ & $<0.001$ \\
\hline $\begin{array}{l}\text { Blood eosinophil count }{ }^{\#} \times 10^{9} \text { cells } \cdot \mathrm{L}^{-1} \\
\text { mean } \pm \mathrm{SD}\end{array}$ & $0.17 \pm 0.21$ & $0.15 \pm 0.14$ & $0.13 \pm 0.17$ & $<0.001$ \\
\hline \multicolumn{5}{|l|}{ Treatment ${ }^{\pi}$} \\
\hline Short-acting $\beta$-agonists & $112(31.6)$ & $169(39.1)$ & 98 (2.3) & $<0.001$ \\
\hline Long-acting $\beta$-agonists & $130(36.7)$ & $260(60.2)$ & $23(0.5)$ & $<0.001$ \\
\hline Long-acting muscarinergic antagonists & $17(4.8)$ & $200(46.3)$ & $10(0.2)$ & $<0.001$ \\
\hline Inhaled corticosteroids & 178 (50.3) & $187(43.3)$ & $40(0.9)$ & $<0.001$ \\
\hline Leukotriene receptor antagonists & $42(11.9)$ & $12(2.8)$ & $0(0)$ & $<0.001$ \\
\hline Antihistamines & $67(18.9)$ & $47(10.9)$ & $261(6.0)$ & $<0.001$ \\
\hline Systemic corticosteroids & $41(11.6)$ & $90(20.8)$ & $156(3.6)$ & $<0.001$ \\
\hline
\end{tabular}

Data are presented as $n(\%)$ unless otherwise stated. Total $n=5104$. IQR: interquartile range. \#: out of the total population, 2923 patients with COVID-19 had blood eosinophil counts within the last 5 years.

१: frequency of different types of medication from the last 12 months were estimated; if a patient received combination therapy, for example inhaled corticosteroids and a long-acting $\beta$-agonist, the medications were split up into their generic forms; treatment with systemic corticosteroids was defined as any prescription filled within the last 12 months. 
compared with the other groups. Among patients with asthma, 217 out of 354 (61.3\%) were women, while patients with COPD and patients without obstructive lung disease showed no obvious differences in sex distribution. In total, 913 out of 5104 (17.9\%) were immigrants or descendants of immigrants.

Only 73 out of 432 (16.9\%) COPD patients were under 65 years of age, while 291 out of $354(82.2 \%)$ patients with asthma were younger than 65 years. In comparison, only 33 out of 354 (9.3\%) were 75 years or older, while among the patients with COPD, 241 out of 432 (55.8\%) were 75 years or older.

We estimated use of respiratory treatments within the last 12 months among patients with asthma and COPD split into each generic pharmaceutical substance. In patients with asthma, inhaled corticosteroids of any kind were used by 178 out of 354 patients (50.3\%). Compared with patients with COPD, use of leukotriene receptor antagonists, antihistamines and inhaled corticosteroids were more common among patients with asthma. Use of systemic corticosteroids, long-acting $\beta$-agonists and long-acting muscarinergic antagonists were more common among patients with COPD compared with patients with asthma. In patients without asthma or COPD, 171 out of 4318 (3.4\%) had received inhalation therapy of any kind within the last 12 months.

We estimated the standardised absolute risk of the severe combined end-point within the first 30 days (figure 2). At day 30, the risk of developing the combined end-point was $21.2 \%$ (95\% CI $18.8-23.6$ ) in patients with COPD; $18.5 \%$ (95\% CI 14.3-22.7) in patients with asthma and $17.2 \%$ (95\% CI 16.1-18.3) in patients with no asthma or COPD. Patients with COPD had significantly higher risk of developing the combined end-point than patients without asthma or COPD with a risk difference of $4.0 \%$ (95\% CI 1.3-6.6; $\mathrm{p}=0.003)$. There was no significant risk difference for patients with asthma compared with patients without asthma or COPD (1.3\%, 95\% CI -3.1-5.6; $\mathrm{p}=0.57)$.

We calculated standardised risks of the individual components of the combined end-point. We found that patients with COPD had significantly higher risk of being diagnosed with "severe COVID-19" compared with patients without obstructive lung diseases with a risk difference of $4.7 \%$ (95\% CI 1.8-7.6; $\mathrm{p}=0.001$ ). For patients with asthma, the risk difference was $2.1 \%(95 \%$ CI $-2.0-6.1 ; p=0.32)$. Risk of death was higher among patients with COPD compared with patients without asthma or COPD (1.9\%, 95\% CI 0.1-3.6; $\mathrm{p}=0.035$ ), while patients with asthma did not have increased risk. We observed no differences in risk of admission to ICU between the disease groups.

We calculated standardised risk for the combined outcome at day 30 after diagnosis of COVID-19 assuming that a patient could have any age between 30 and 100 years (figure 3). At age 30 years, patients without obstructive lung disease had a mean risk of $2.9 \%$ (95\% CI 1.8-3.9), patients with asthma had a mean risk of 3.2\% (95\% CI 0.7-5.8) and patients with COPD had a standardised risk of 14.1\% (95\% CI 2.3-25.8). At age 50 years, the risk increased to $10.1 \%$ (95\% CI 8.6-11.5) for patients without lung disease, $14.5 \%$ (95\% CI 6.7-22.3) for patients with asthma and 21.0\% (95\% CI 9.9-32.2) for patients with COPD. At age 70, the risk of the combined outcome further increased to $29.2 \%$ (95\% CI 21.3-37.2) for patients without lung disease, 46.6\% (95\% CI 14.7-78.5) for patients with asthma and 33.0\% (95\% CI 25.3-40.7) for patients with COPD. Age-standardised risks of the individual components of the combined outcome of severe COVID-19 were estimated (figure 4). At age 70, patients with COPD had a significantly lower risk of being admitted to an ICU compared with patients with asthma and those with no lung disease $(\mathrm{p}<0.001)$ (figure 5). Risk of death increased with age in all disease groups. Standardised absolute risks at specific age points with confidence intervals are supplied in the supplementary material (Supplementary 5).

\section{Eosinophilic inflammation}

In the total study population, 2923 out of 5104 had measured blood eosinophil levels within the last 5 years. In a complete-case analysis, we estimated the standardised absolute risk at 30 days after diagnosis of COVID-19 in four groups: asthma, COPD, asthma/COPD combined and no asthma/COPD (figure 6). In patients with COPD, low counts of eosinophils in blood were associated with increased risk of the

FIGURE 2 Prevalence of COPD and asthma as a percentage of the sample population versus the general population on 10 May 2020 when using the same definition as in our sample population. COVID-19: coronavirus disease 2019.
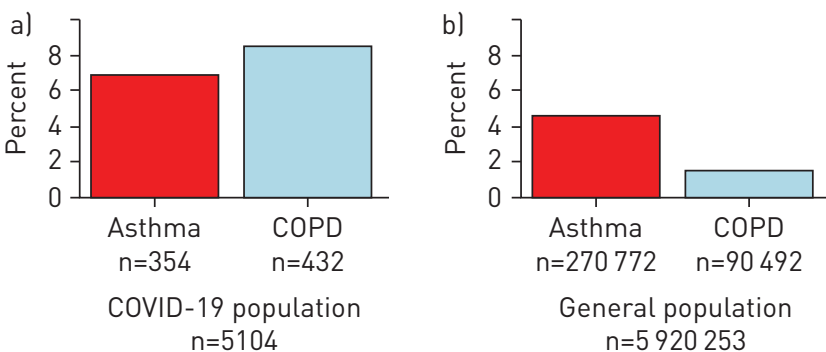


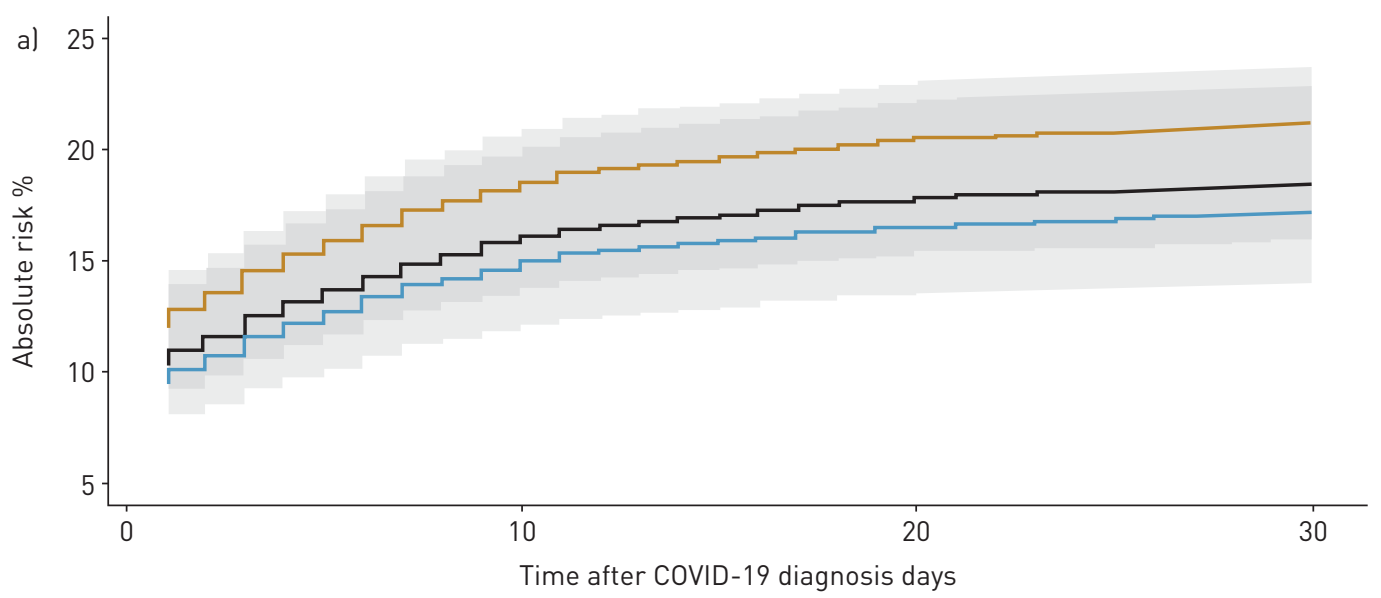

$95 \%$ confidence band

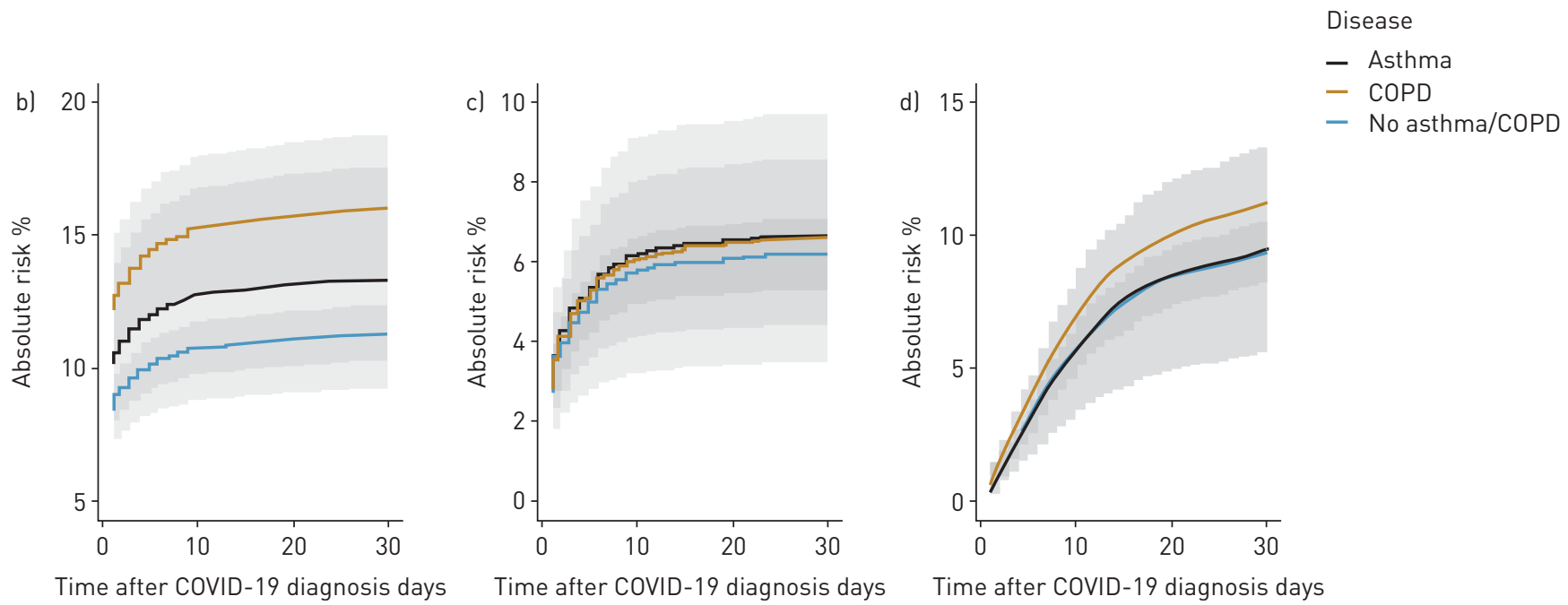

FIGURE 3 a) Standardised absolute risk of combined end-point for coronavirus 2019 (COVID-19) in the first 30 days after diagnosis. The lower panel shows the individual parts of the combined end-points. a) Severe COVID-19; b) intensive care unit admission; c) death. ICU: intensive care unit.

combined outcome with a risk difference of $18.5 \%$ (95\% CI 5.9-31.1; $\mathrm{p}=0.002$ ). The same pattern with high eosinophils being protective against severe disease was seen when patients with asthma and COPD were combined (risk difference 13.6\%; 95\% CI 4.3-22.9; $\mathrm{p}=0.002$ ).

\section{Discussion}

In this retrospective cohort study of Danish COVID-19 patients with asthma, COPD or no obstructive lung disease, we found that patients with COPD had a slightly increased risk of a severe combined

FIGURE 4 Standardised absolute risk showing the relationship between type of chronic disease and risk of the combined end-point of coronavirus disease 2019 (COVID-19) at day 30 between age 30 and 100 years. The combined end-point is a combination of death, severe respiratory syndrome in COVID-19 by International Classification of Diseases (ICD)-10 codes and admission to an intensive care unit.

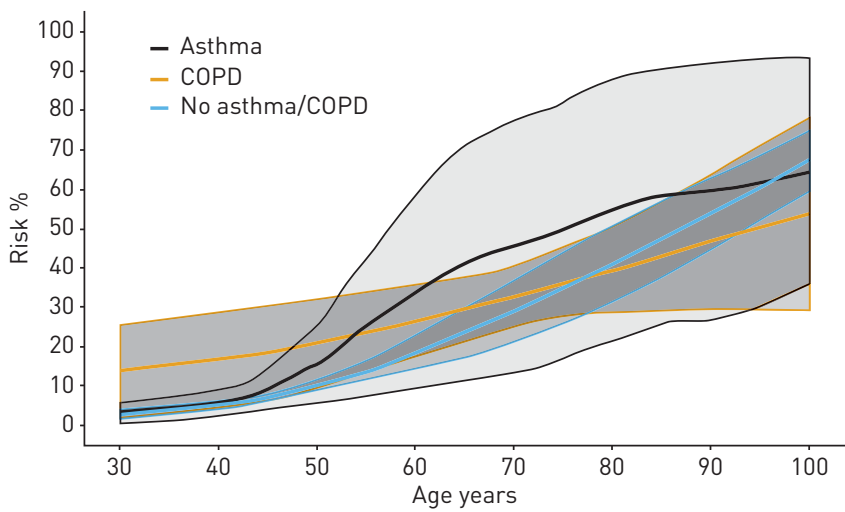



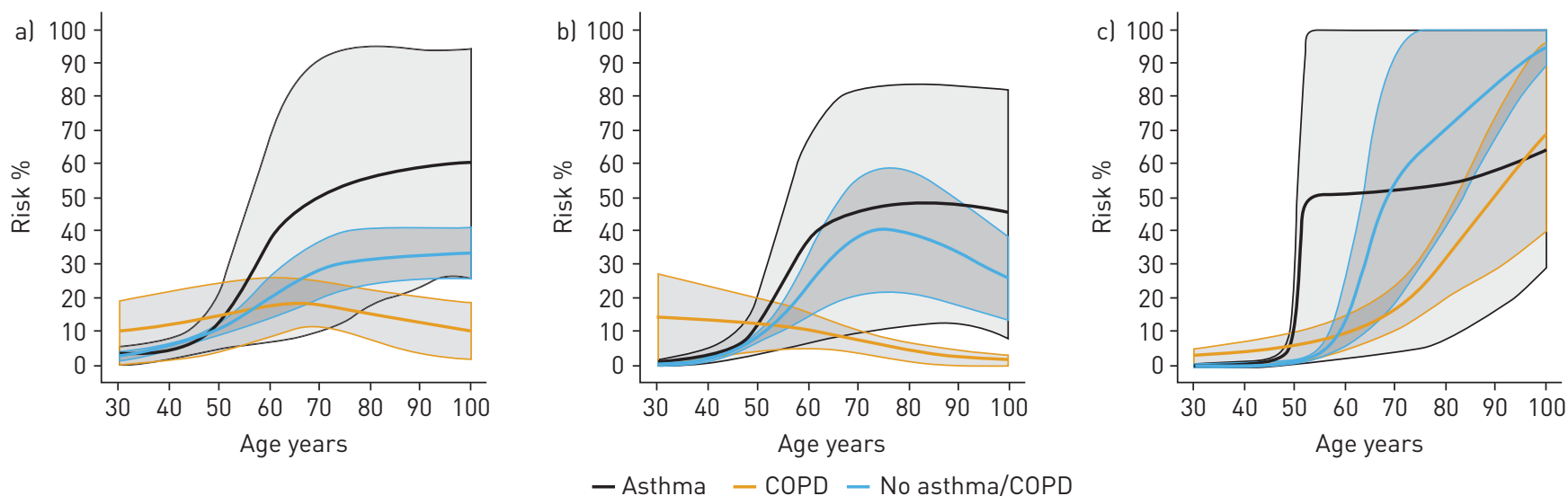

FIGURE 5 Components of the standardised risk of the combined outcome of coronavirus disease 2019 (COVID-19) at 30 days by age. Displayed is the absolute risk of the individual components of the combined outcome standardised by age. al Severe COVID-19; b) intensive care unit admission; c) death.

outcome of COVID-19, while patients with asthma showed no increased risk. When the analyses were standardised by age, the risk differences disappeared. Compared with the general population, patients with asthma and COPD were over-represented suggesting that they could be more susceptible to COVID-19 requiring hospitalisation or medical assistance. Lastly, we found that low counts of blood eosinophils were associated with worse outcomes of COVID-19 in patients with COPD.

Patients with COPD had increased absolute risk of severe COVID-19. However, in age-standardised analysis, there were no significant differences in risk between patients with asthma, COPD or no obstructive lung disease suggesting that asthma and COPD might not be independent risk factors for

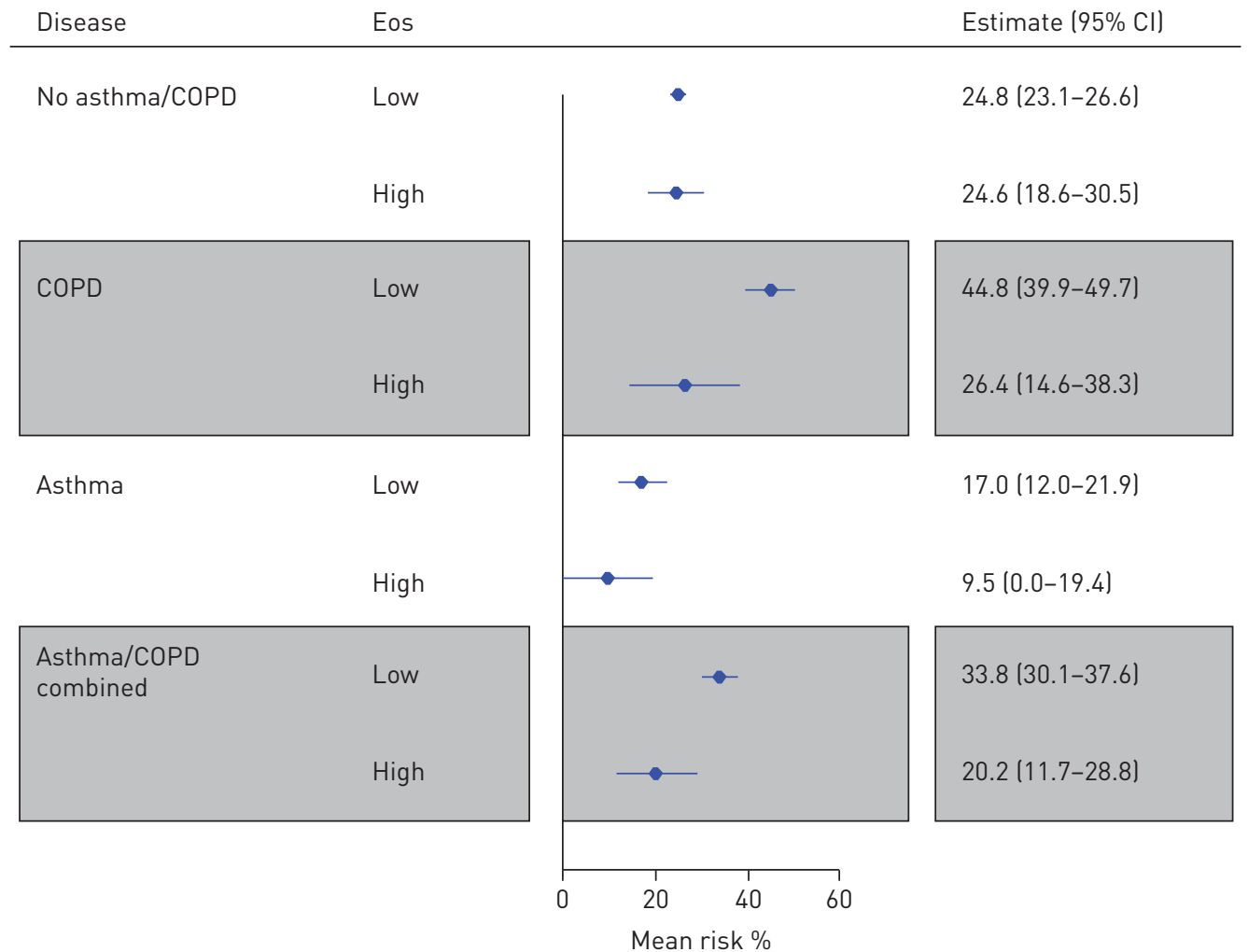

FIGURE 6 Risk of severe outcomes of coronavirus disease 2019 (COVID-19) and blood eosinophil values. Displayed are the standardised absolute risks of the combined outcome of COVID-19 given that a patient had low or high levels of blood eosinophils $\left(<0.3 \times 10^{9}\right.$ or $\geqslant 0.3 \times 10^{9}$ cells. $\left.\mathrm{L}^{-1}\right)$. The estimations displayed are the risks at day 30 after first diagnosis of COVID-19. 
adverse outcomes of COVID-19. Further, patients with COPD were less likely to be admitted to an ICU. This is probably because of the presence of other comorbid conditions, which indicates that their recovery potential is low. However, as the primary outcome in this study was a combined outcome of three COVID-19 outcomes, patients with COPD were still at risk of severe COVID-19 and death.

We observed no significant differences in age-standardised risk for the combined severe COVID-19 outcome between the disease groups. However, in patients with asthma at age 70 the standardised risk for the combined outcome at day 30 increased dramatically to $46.6 \%$, but as the confidence intervals are broad because of the low incidence of COVID-19 in Denmark, this difference was not significant. Further studies investigating outcomes of patients with asthma are warranted to determine whether they are at higher risk of severe outcomes of COVID-19.

Interestingly, we found that blood eosinophils were associated with disease outcome among patients with COVID-19 and COPD. It is notable that low levels of eosinophils were associated with worse outcomes of COVID-19, since high levels of eosinophilia are associated with detrimental effects in COPD. It is therefore imperative that the mechanisms and relationships between Type 2 inflammation and outcomes of COVID-19 are assessed in future prospective studies among patients with obstructive lung diseases.

When applying our definition of asthma and COPD to the general population, we observed a relatively low number of patients with asthma and COPD. A reason for this could be that we did not include short-acting $\beta$-agonists as a diagnostic criterion for asthma and COPD, since it is difficult to determine whether it was initiated due to asthma, COPD or other reasons.

It is surprising that only $50 \%$ of the population with asthma had filled prescriptions with inhaled corticosteroids within the last 12 months, as inhaled corticosteroids are considered the cornerstone treatment in asthma. This raises questions as to whether they are former asthma patients or whether it is a matter of poor adherence. From a pulmonary perspective the latter seems likely, and it is possible that this could be associated with their high admission rate [18]. However, it could be that patients with asthma are more aware of their respiratory symptoms and thus are more likely to seek medical care.

A strength of our study is that we were able to include all patients registered in the Danish registers diagnosed with COVID-19. The Danish healthcare system is tax-financed with free universal access and is linked with a personal identification number. This enabled us to connect a patient's COVID-19 diagnosis to socioeconomic and medical data before the specific outcome. Therefore, our description of the included population is precise, objective and generalisable.

Our study is limited by its observational nature, and as with all register-based studies, we are reliant on precise data from clinicians and healthcare systems nationwide. This means that our diseases of interest (asthma and COPD) could have been both over- and underdiagnosed. Further, we had no access to data on degree of airflow limitation and thus, severity of COPD and asthma could only be determined by medication use. In our study, only patients registered with COVID-19 in a hospital setting were included. This means that patients in this study probably have a higher burden of disease and comorbidities compared with COVID-19 patients outside the hospital system. However, during the current pandemic clear guidelines on reporting COVID-19 and related outcomes were established, and this makes us believe that the diagnoses and reporting of severe COVID-19 are precise [19].

In conclusion, patients with COPD had slightly increased standardised absolute risk of a combined outcome of COVID-19 compared with patients without asthma and COPD. However, in age-standardised analysis, the risk difference disappeared. This suggests that asthma and COPD are not independent risk factors for adverse outcomes of COVID-19. However, patients with asthma and COPD were over-represented in our population, suggesting that patients with pulmonary disease are more susceptible to COVID-19 demanding hospitalisation, and this should be investigated further.

Acknowledgements: The Centre for Physical Activity Research (CFAS) is supported by TrygFonden (grants ID 101390 and ID 20045). The authors wish to express their gratitude to Jørn Korsbø Pedersen and his colleagues at Statistics Denmark for their help regarding data access.

Author contributions: E.S.H. Hansen, A.L. Moeller, C. Torp-Pedersen and V. Backer initiated the study. E.S.H. Hansen and A.L. Moeller produced the initial manuscript. E.S.H. Hansen, A.L. Moeller and C. Torp-Pedersen made the initial analyses. All authors contributed to the interpretation of the data and manuscript writing. All authors approved the final manuscript before submitting for publication. E.S.H. Hansen and C. Torp-Pedersen take full responsibility for the results. E.S.H. Hansen and C. Torp-Pedersen affirm that this manuscript is an honest, accurate and transparent account of the study being reported; that no important aspects of the study have been omitted; and that any discrepancies from the study as planned have been explained.

Conflict of interest: E.S.H. Hansen has nothing to disclose. A.L. Moeller has nothing to disclose. V. Backer reports grants and personal fees from AstraZeneca, GSK, TEVA, Chiesi, Sanofi, MSD and Novartis, outside the submitted work. 
M.P. Andersen has nothing to disclose. L. Kober reports personal fees from Novo Nordisk, Boehringer, AstraZeneca and Novartis outside the submitted work. K. Kragholm reports personal fees from Novartis outside the submitted work.

C. Torp-Pedersen reports grants from Novo Nordisk and Bayern outside the submitted work.

\section{References}

1 Ntritsos G, Franek J, Belbasis L, et al. Gender-specific estimates of COPD prevalence: a systematic review and meta-analysis. Int J Chron Obstruct Pulmon Dis 2018; 13: 1507-1514.

2 Hosseini M, Almasi-Hashiani A, Sepidarkish M, et al. Global prevalence of asthma-COPD overlap (ACO) in the general population: a systematic review and meta-analysis. Respir Res 2019; 20: 4-13.

3 Wedzicha JA, Seemungal TA. COPD exacerbations: defining their cause and prevention. Lancet 2007; 370: 786-796.

$4 \quad$ Rabe KF, Watz H. Chronic obstructive pulmonary disease. Lancet 2017; 389: 1931-1940.

5 Guan WJ, Liang WH, Zhao Y, et al. Comorbidity and its impact on 1590 patients with Covid-19 in China: a nationwide analysis. Eur Respir J 2020; 55: 2000547.

6 Docherty AB, Harrison EM, Green CA, et al. Features of 16,749 hospitalised UK patients with COVID-19 using the ISARIC WHO Clinical Characterisation Protocol. medRxiv 2020; preprint [https://doi.org/10.1101/2020.04.23. 20076042].

7 Bafadhel M, Peterson S, De Blas MA, et al. Predictors of exacerbation risk and response to budesonide in patients with chronic obstructive pulmonary disease: a post-hoc analysis of three randomised trials. Lancet Respir Med 2018; 6: 117-126.

8 Chhiba KD, Patel GB, Vu THT, et al. Prevalence and characterization of asthma in hospitalized and nonhospitalized patients with COVID-19. J Allergy Clin Immunol 2020; 146: 307-314.

9 Schmidt M, Schmidt SAJ, Sandegaard JL, et al. The Danish National patient registry: a review of content, data quality, and research potential. Clin Epidemiol 2015; 7: 449-490.

10 Backer V, Lykkegaard J, Bodtger U, et al. The Danish national database for asthma. Clin Epidemiol 2016; 8 : 601-606.

11 Andersen PK, Gill RD. Cox's regression model for counting processes: a large sample study. Ann Stat 1982; 10: $1100-1120$.

12 Hernán M, Robins J. Causal Inference - Part 2. Boca Raton, Chapman \& Hall/CRC, 2018.

13 COPD Global Initiative. 2020 Report. Global Initiative for Chronic Obstructive Lung Disease. 2020. https:// goldcopd.org/wp-content/uploads/2019/11/GOLD-2020-REPORT-ver1.0wms.pdf Date last accessed: May 19, 2020.

14 Eisner MD, Blanc PD, Omachi TA, et al. Socioeconomic status, race and COPD health outcomes. J Epidemiol Community Health 2011; 65: 26-34.

15 Greenland S, Pearl J, Robins JM. Causal diagrams for epidemiologic research. Epidemiology 1999; 10: 37-48.

16 Textor J, van der Zander B, Gilthorpe MS, et al. Robust causal inference using directed acyclic graphs: The R package "dagitty". Int J Epidemiol 2016; 45: 1887-1894.

17 R Core Team. R: A language and Environment for Statistical Computing. 2019. www.r-project.org/.

18 Mäkelä MJ, Backer V, Hedegaard M, et al. Adherence to inhaled therapies, health outcomes and costs in patients with asthma and COPD. Respir Med 2013; 107: 1481-1490.

19 Fosbøl EL, Butt JH, Østergaard L, et al. Association of angiotensin-converting enzyme inhibitor or angiotensin receptor blocker use with COVID-19 diagnosis and mortality. JAMA 2020; 324: 168-177. 